Document downloaded from:

http://hdl.handle.net/10251/155062

This paper must be cited as:

Rubio Arjona, L.; Rodrigo Peñarrocha, VM.; Molina-García-Pardo, JM.; Juan Llacer, L.; Pascual Garcia, J.; Reig, J.; Sanchis Borrás, C. (2019). Millimeter Wave Channel Measurements in an Intra-Wagon Environment. IEEE Transactions on Vehicular Technology. 68(12):12427-12431. https://doi.org/10.1109/TVT.2019.2947205

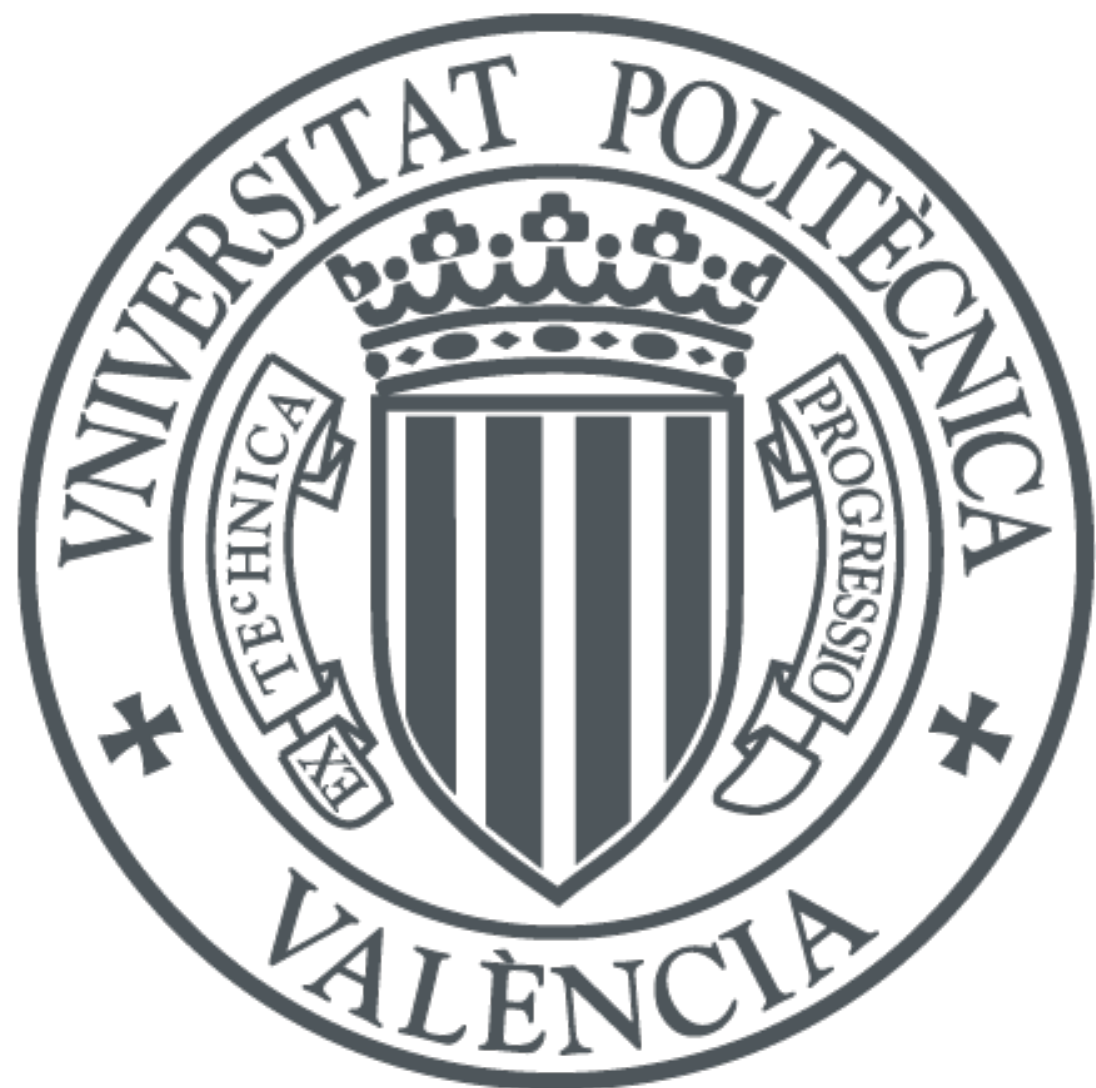

The final publication is available at

https://doi.org/10.1109/TVT.2019.2947205

Copyright Institute of Electrical and Electronics Engineers

Additional Information

(C) 2019 IEEE. Personal use of this material is permitted. Permissíon from IEEE must be obtained for all other uses, in any current or future media, including reprinting/republishing this material for advertisíng or promotional purposes, creating new collective works, for resale or redistribution to servers or lists, or reuse of any copyrighted component of this work in other works." 


\title{
Millimeter Wave Channel Measurements in an Intra-Wagon Environment
}

\author{
Lorenzo Rubio, Senior Member, IEEE, Vicent M. Rodrigo Peñarrocha, José-María Molina-García-Pardo, Leandro \\ Juan-Llácer, Senior Member, IEEE, Juan Pascual-García, Juan Reig, Senior Member, IEEE, and Concepción \\ Sanchis-Borras
}

\begin{abstract}
In this correspondence, useful measurement results of the propagation channel characteristics in an intra-wagon environment at millimeter wave (mmWave) frequencies are presented. The measurements were collected inside an underground convoy from 25 to $40 \mathrm{GHz}$ in the frequency domain. A broadband radio over fiber (RoF) link was used in order to avoid the high losses introduced by cables at these frequencies, thus allowing long distances between the transmitter and receiver antennas. Values of the path loss exponent and delay spread are reported at the potential 26,28 and $38 \mathrm{GHz}$ bands to deploy the future fifth-generation (5G) systems. These results allow us to have a better knowledge of the path loss and time dispersion characteristics of the propagation channel in this particular environment, characterized by rich-scattering with long delays.
\end{abstract}

Index Terms-MmWave, intra-wagon, 5G, channel measurements, path loss, time dispersion.

\section{INTRODUCTION}

$\mathbf{M}$ OBILE communication networks in future railways should be designed to provide both high-capacity and high-data rate in order to offer broadband services [1]. In addition to the traditional sub- $6 \mathrm{GHz}$ bands, the millimeter wave (mmWave) band has been proposed to enable such broadband services in train applications [2]. In the last World Radio Communication Conference (WRC) of the International Telecommunication Union (ITU), held in 2015 , the $26 \mathrm{GHz}$ $(24.25-27.5 \mathrm{GHz})$ and $38 \mathrm{GHz}(37-40.5 \mathrm{GHz})$ bands were fixed to locate the future fifth-generation (5G) systems, on a primary basis, above $24 \mathrm{GHz}$ [3]. The final decision will be determined at the next WRC in 2019. Furthermore, the $28 \mathrm{GHz}(27.5-28.35 \mathrm{GHz})$ band has also been licensed for mobile applications in USA.

In [1] and [2], at least five future railway service scenarios have been defined, including: train-to-infrastructure, inter-

This work has been funded in part by the Ministerio de Economía, Industria y Competitividad of the Spanish Government under the national projects TEC2016-78028-C3-2-P and TEC2017-86779-C2-2-R, through the Agencia Estatal de Investigación (AEI) and the Fondo Europeo de Desarrollo Regional (FEDER).

L. Rubio, V. M. Rodrigo Peñarrocha and J. Reig are with the iTEAM Research Institute, Universitat Politècnica de València, 46022 Valencia, Spain (email: \{lrubio; vrodrigo; jreigp $\} @$ dcom.upv.es).

J. M. Molina-García-Pardo, J. Pascual-García and L. Juan-Llácer are with the Information Technologies and Communications Department, Universidad Politécnica de Cartagena, 30202 Cartagena, Murcia, Spain (email: \{josemaria.molina; juan.pascual; leandro.juan\}@upct.es).

C. Sanchis-Borras is with the Department of Technical Sciences, Universidad Católica San Antonio de Murcia (UCAM), 30107 Murcia, Spain (email: csanchis@ucam.edu).

Copyright @ 2015 IEEE. Personal use of this material is permitted. However, permission to use this material for any other purposes must be obtained from the IEEE by sending a request to pubs-permissions@ieee.org wagon, intra-wagon, inside-the-station, and infrastructure-toinfrastructure. There have been many recent studies on the mmWave band involved in both outdoor [2], [4]-[6], and indoor railway scenarios [6], [7]. In [2], channel measurements for inter-wagon communications have been performed at $64.5 \mathrm{GHz}$. In [4], channel measurements have been carried out in a train-to-infrastructure scenario at $60 \mathrm{GHz}$. In [5], the authors have analyzed several propagation parameters using a 3D ray tracer in a train-to-infrastructure scenario at $25.25 \mathrm{GHz}$. In [6], the influence of typical railway objects in the propagation channel has been investigated using ray tracing techniques. However, with the exception of [7], there are no published works in which some aspects of the propagation channel in intra-wagon environments are analyzed from real measurements. In [7], channel measurements have been performed in the $26.5-40 \mathrm{GHz}$ band using directive antennas. The authors have mainly investigated the shape of the power delay profile by establishing a cluster identification, but no results on path loss and delay spread values are reported at different frequencies. Therefore, more channel measurements and efforts are necessary to have a better knowledge of the path loss and time dispersion in intra-wagon environments, with special interest in the $25-40 \mathrm{GHz}$ band and using omnidirectional antennas.

Thus, this correspondence provides novel measurement results on path loss and time dispersion in an intra-wagon environment. A channel sounder based on a vector network analyzer (VNA) has been used to carry out the channel measurements. A broadband radio over fiber (RoF) link was also used to increase the distance between the transmitter (Tx) and the receiver $(\mathrm{Rx})$.

\section{Channel Measurements}

\section{A. Propagation Environment}

The measurement campaign was carried out inside an underground convoy, parked in the garages of Ferrocarriles de la Generalitat Valenciana (FGV) in Valencia, Spain. The convoy consists of four wagons connected forming a single space. The convoy has a small cabinet for the driver on both ends, separated from the passengers. The total interior dimensions of the convoy are 55.25-m-long, 2.55-m-width and 2.15-m-high.

There are many handholds, side seats and other furniture in the convoy. The seats are resin molded, reinforced with glass fiber, and both the handholds and the roof are made of stainless steel. The floor is made of stratified rubber, the 


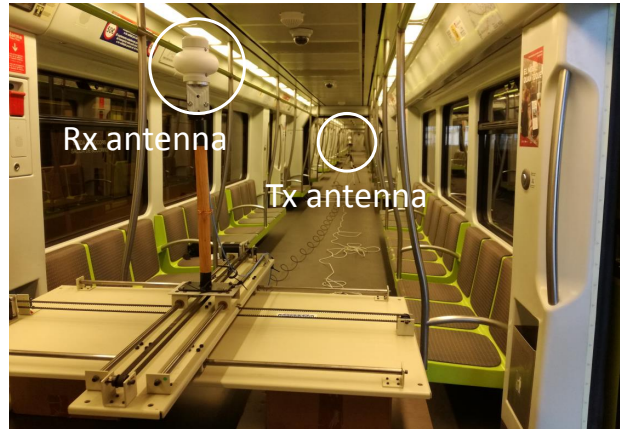

Fig. 1. Interior view of the convoy with the Tx and Rx antennas.

windows are made of laminated glass and the doors are of aluminum and glass. Fig. 1 shows the interior view of the convoy. The presence of numerous metallic elements in the structure of the convoy makes it a rich-multipath environment.

\section{B. Measurement Procedure and Setup}

The complex channel transfer function (CTF), denoted by $H(f)$, has been measured in the frequency domain using the Keysight N5227A VNA. The QOM-SL-0.8-40-K-SG-L ultrawideband omnidirectional antennas, developed by Steatite Ltd company, have been used at the $\mathrm{Tx}$ and $\mathrm{Rx}$ sides. These antennas operate from $800 \mathrm{MHz}$ to $40 \mathrm{GHz}$, and have vertical polarization. Fig. 2 shows the measured gain in our anechoic chamber (square marker) and the cubic spline interpolation gain in the $25-40 \mathrm{GHz}$ frequency band in the horizontal plane. The $3 \mathrm{~dB}$ beamwidth of the antennas in the elevation plane, also known as half power beamwidth (HPBW), ranges from $20^{\circ}$ to $40^{\circ}$ in the band of interest, and the $10 \mathrm{~dB}$ beamwidth ranges from $45^{\circ}$ to $100^{\circ}$.

The Tx subsystem has been connected to the VNA through an amplified (with $35 \mathrm{~dB}$ gain) broadband RoF link (Optica OTS-2 mode ${ }^{1}$ developed by Emcore) to avoid the high losses of cables at mmWave frequencies, thus increasing the dynamic range in the measurement. This increase in the dynamic range allows us to use omnidirectional antennas due to the fact that relevant radio channel characteristics, such as delay spread, could be affected by the use of non-omnidirectional or directive antennas [8].

The Rx antenna has been located in a $\mathrm{XY}$ positioning system, implementing a $7 \times 7$ uniform rectangular array (URA). This means that for each Tx location, a total of 49 $(7 \times 7)$ CTFs have been measured. The separation of the URA elements has been $3.04 \mathrm{~mm}$ (less than $\lambda / 2=3.7 \mathrm{~mm}$ at $40 \mathrm{GHz})$. Both the VNA and the XY positioning system have been controlled by a personal computer, measuring the $S_{21}(f)$ scattering parameter directly from 25 to $40 \mathrm{GHz}$, equivalent to the CTF of the propagation channel, i.e., $H(f) \equiv S_{21}(f)[9]$. Before the measurements, the channel sounder was calibrated carefully. A response calibration process was performed by disconnecting the $\mathrm{Tx}$ and $\mathrm{Rx}$ antennas and connecting the

\footnotetext{
${ }^{1}$ These RoF links are used to construct transparent inter- and intra-facility links for RF and microwave signal transport and antenna remoting, among others.
}

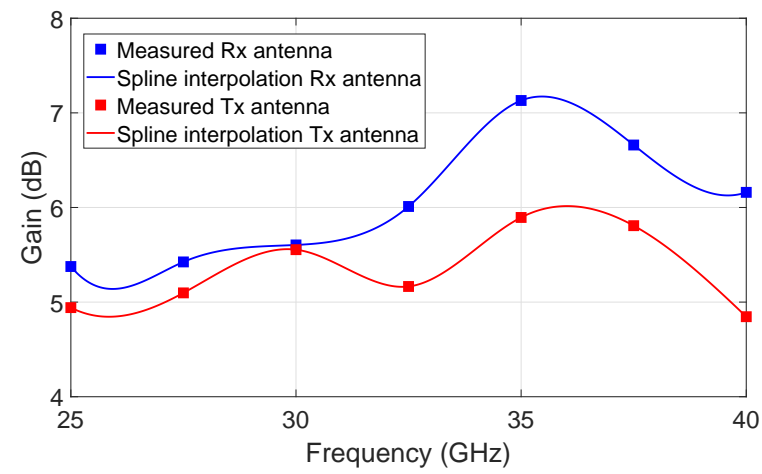

Fig. 2. Measured and cubic spline interpolation antennas gain from 25 to $40 \mathrm{GHz}$ in the horizontal plane.

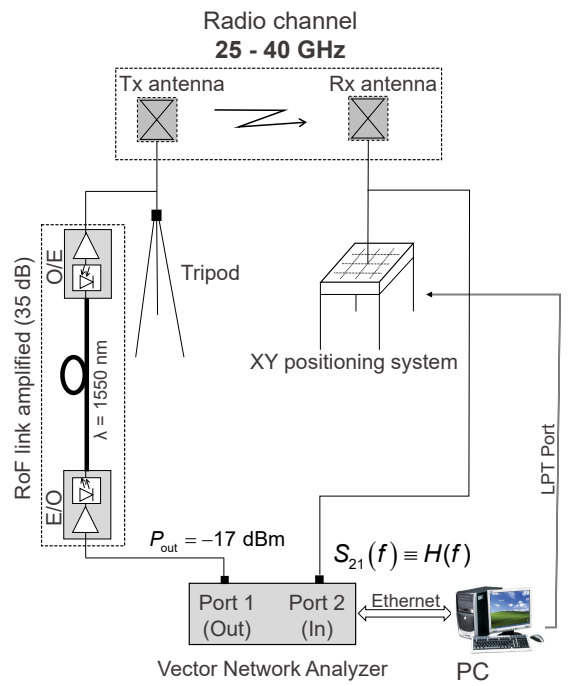

Fig. 3. Schematic diagram of the channel sounder.

transmitter cable output (Tx antenna connector) and receiver cable input ( $\mathrm{Rx}$ antenna connector). The calibration process moves the time reference points from the VNA ports to the calibration points. Thus, the measured CTF takes into account the joint response of the propagation channel and the Tx and Rx antennas, also known as the radio channel [10]. Notice that due to their dimensions, the delay introduced by the antennas themselves are insignificant. A schematic diagram of the channel sounder is illustrated in Fig. 3

During the measurements, the RF signal level at the VNA output has been $-17 \mathrm{dBm}$ in order to not saturate the amplifier at the input of the electro-optical converter in the RoF link. The CTF has been measured using 8192 frequency points, avoiding temporal aliasing, that is a frequency resolution of about $\Delta f=1.83 \mathrm{MHz}$, which corresponds to a maximum unambiguous excess delay estimated as $1 / \Delta f$ of $546 \mathrm{~ns}$, and a maximum observable distance calculated as $c_{0} / \Delta f$, with $c_{0}$ the speed of light, of about $164 \mathrm{~m}$. The bandwidth of the intermediate frequency (IF) filter at the VNA, denoted by $B_{\mathrm{IF}}$, conditions both the thermal noise level at the $\mathrm{Rx}$ and the measurement acquisition (sweep) time. Thus, a low value of $B_{\mathrm{IF}}$ reduces the power level of the noise floor and 


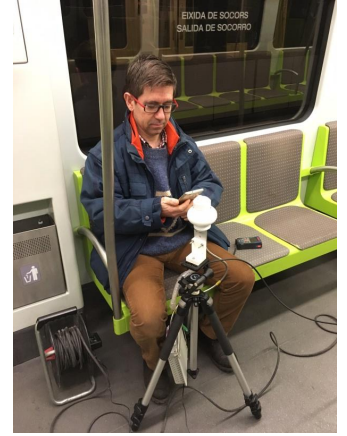

Fig. 4. View of a Tx antenna location in Scenario 2.

therefore increases the dynamic range in the measurement, allowing us to perform channel measurements with a greater Tx-Rx distance. Nevertheless, low values of $B_{\mathrm{IF}}$ increase the acquisition time. As a compromise between acquisition time and dynamic range in the measurement, the $B_{\mathrm{IF}}$ was set to $100 \mathrm{~Hz}$. As a reference, in [11] the authors used $500 \mathrm{~Hz}$ in indoor office channel measurements at mmWave frequencies.

In our measurements, the Tx antenna has been located manually in different locations along the convoy, imitating the position of a user equipment (UE), e.g., a smartphone or tablet. The Rx subsystem remained fixed in the same location, next to the cabin in the center of the wagon, imitating the position of an access point (AP) that serves the passengers inside the convoy. Fig. 1 shows the position of the Rx antenna, mounted on the XY positioning system, and the Tx antenna located in the center of the convoy. To guarantee stationary channel conditions, the convoy was parked in the FGV garages, away from other convoys and staff, and the doors of the convoy remained closed to prevent people from entering during the acquisition time ( 2 hours per Tx position).

The measurements have been collected under line-of-sight (LoS) conditions. The Rx antenna height was $1.63 \mathrm{~m}$ with respect to the floor level. Based on the Tx antenna height and its location, two scenarios have been defined:

- Scenario 1: The Tx antenna has been located in the center of the convoy (see Fig. 1p at a height of $1.45 \mathrm{~m}$ with respect to the floor, simulating the position of a UE used by a passenger who travels standing. Eight $\mathrm{Tx}$ antenna locations have been measured with a Tx-Rx distance from 5 to $50 \mathrm{~m}(5,10,15,20,35,40,45$ and $50 \mathrm{~m})$.

- Scenario 2: The Tx antenna has been located next to the seats at a height of $0.85 \mathrm{~m}$ with respect to the floor, simulating the position of a UE used by a passenger who travels seated (see Fig. 4). Six Tx antenna locations have been measured with a Tx-Rx distance from 5 to $50 \mathrm{~m}$ (5, $12,20,40,45$ and $50 \mathrm{~m}$ ).

\section{iII. Measurement Results}

For each frequency band, the path loss and the temporal dispersion introduced by the propagation channel have been obtained over a bandwidth of $2 \mathrm{GHz}$ (maximum temporal resolution of $0.5 \mathrm{~ns}$ ), being 26,28 and $38 \mathrm{GHz}$ the central frequencies.
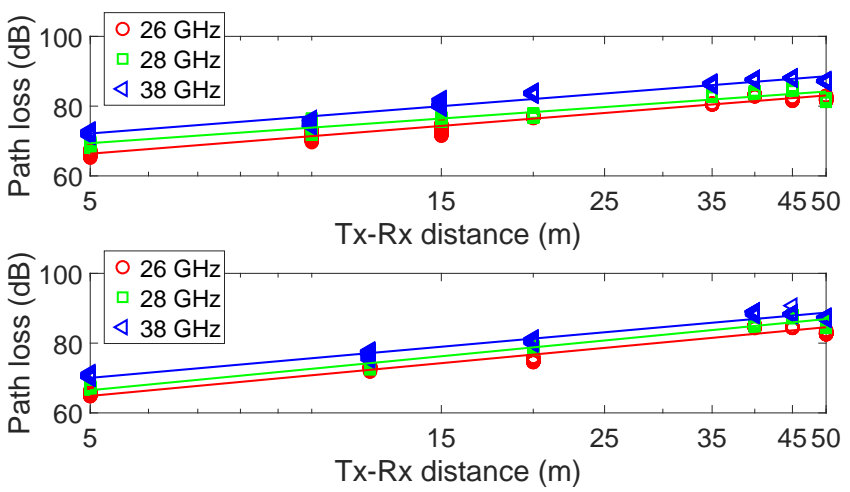

Fig. 5. Path loss in terms of the Tx-Rx distance, in logarithmic scale, in Scenario 1 (up) and Scenario 2 (down).

\section{A. Path Loss}

For each position of the Rx antenna in the URA, the path loss in logarithmic units, $P L$, can be derived from the CTF by:

$$
P L(d)=-10 \log _{10}\left(\frac{1}{N} \sum_{n=1}^{N} \frac{\left|H\left(f_{n}, d\right)\right|^{2}}{g_{\mathrm{Tx}}\left(f_{n}\right) g_{\mathrm{Rx}}\left(f_{n}\right) M\left(f_{n}\right)}\right),
$$

where $d$ refers to the distance between the Tx and the center of the URA for each Tx location, indicated simply as TxRx distance; $N=1091$ is the number of frequency samples considered in each frequency band; $f_{n}$ is the $\mathrm{n}$ - $t h$ frequency sample; $g_{\mathrm{Tx}}\left(f_{n}\right)$ and $g_{\mathrm{Rx}}\left(f_{n}\right)$ are the gain of the Tx and $\mathrm{Rx}$ antennas, respectively, in the direction defined by the LoS contribution; and the term $M\left(f_{n}\right)$ takes into account the mismatch of the antennas $2, M\left(f_{n}\right)$ is calculated by:

$$
M\left(f_{n}\right)=\left(1-\left|S_{11}^{\mathrm{Tx}}\left(f_{n}\right)\right|^{2}\right)\left(1-\left|S_{11}^{\mathrm{Rx}}\left(f_{n}\right)\right|^{2}\right),
$$

being $S_{11}^{\mathrm{Tx}}\left(f_{n}\right)$ and $S_{11}^{\mathrm{Rx}}\left(f_{n}\right)$ the $S_{11}(f)$ scattering parameter of the Tx and Rx antennas, respectively.

Fig. 5 shows the path loss in terms of the Tx-Rx distance in both scenarios at 26, 28 and $38 \mathrm{GHz}$. For each Tx position, the path loss corresponding to the $49 \mathrm{Rx}$ antenna positions in the URA has been depicted.

The floating-intercept (FI) path loss model has been adopted in many works related to mmWave propagation and channel standardizations, e.g. 3GPP channel models [12], [13]. In the FI model, the path loss is given by:

$$
P L^{\mathrm{FI}}(d)=\beta+10 \alpha \log _{10}(d)+\chi_{\sigma},
$$

being $\beta$ the floating-intercept parameter (offset term); $\alpha$ the path loss exponent, related to both the environment and propagation conditions; and $\chi_{\sigma}$ a zero mean Gaussian random variable (in $\mathrm{dB}$ ) with standard deviation $\sigma$, which describes the large-scale signal fluctuations about the mean path loss over

\footnotetext{
${ }^{2}$ The term antenna gain used here corresponds to the definition of the IEEE Standard 145-2013 for definitions of terms for antennas, which does not include the mismatch losses. In ultra-wideband antennas the return loss can be higher than $-10 \mathrm{~dB}$, and in this case it is necessary to take into account the antenna mismatch in the path loss estimation.
} 
TABLE I

Path Loss Model Parameters

\begin{tabular}{l|c|c|c}
\hline \hline Frequency & $\beta\left(\beta_{95 \%}\right)(\mathrm{dB})$ & $\alpha\left(\alpha_{95 \%}\right)$ & $\sigma(\mathrm{dB})$ \\
\hline Scenario 1 & & & \\
$26 \mathrm{GHz}$ & $54.69(54.30-55.80)$ & $1.67(1.64-1.70)$ & 0.96 \\
$28 \mathrm{GHz}$ & $59.04(58.47-59.61)$ & $1.48(1.44-1.52)$ & 1.41 \\
$38 \mathrm{GHz}$ & $60.70(60.24-61.16)$ & $1.64(1.60-1.68)$ & 1.14 \\
\hline Scenario 2 & & & \\
$26 \mathrm{GHz}$ & $51.09(50.45-51.74)$ & $1.97(1.92-202)$ & 1.43 \\
$28 \mathrm{GHz}$ & $52.24(51.77-52.70)$ & $2.04(2.00-2.08)$ & 1.03 \\
$38 \mathrm{GHz}$ & $56.97(56.45-57.49)$ & $1.87(1.83-1.91)$ & 1.15 \\
\hline
\end{tabular}

distance. The path loss fitting results for the FI model are shown in Fig. 5 As can be observed, the FI model exhibits a good fit, although it is worthwhile to note that for Tx-Rx distances larger than $40 \mathrm{~m}$ a constructive interference attributed to the multipath contributions (MPCs) on the direct path (LoS) appears in Scenario 2, similar to the propagation interference in some corridor environments [14]. Table I] summarizes the value of the path loss model parameters derived from the measured path loss in all positions of the $\mathrm{Rx}$ antenna in the URA, using the minimum-mean-square-error (MMSE) approach. The 95\% confidence interval of $\beta$ and $\alpha$ have been also summarized in Table I It is worth noting that these values are lower than 2, which corresponds to the path loss exponent in free space propagation. These values can be explained by the existence of a waveguide effect as a consequence of the elongated structure of the environment. This waveguide effect provokes the MPCs to be guided along the convoy and constructively interfere at the Rx antenna [15]-[17]. This behavior is in agreement with [7] in a similar intra-wagon environment, where a path loss exponent of 1.6 was obtained for a maximum Tx-Rx distance of $17 \mathrm{~m}$. Path loss exponents lower than 2 have been also obtained in other published studies based on channel measurements conducted at frequencies above $6 \mathrm{GHz}$ and mmWave bands in LoS conditions. In [12], path loss exponents equal to 1.1 and 1.3 have been obtained at 28 and $73 \mathrm{GHz}$, respectively, in an indoor environment. In corridor environments, path loss exponents equal to 1.44 and 1.55 have been measured at 10 and $11 \mathrm{GHz}$, respectively, in [18]. Also, path loss exponents of 1.6 and 1.7 have been measured at 14 and $22 \mathrm{GHz}$, respectively, in [14]. At 28 and $38 \mathrm{GHz}$, path loss exponents equal to 0.9 and 1.4, respectively, have been also measured in a corridor environment.

From Table [ the value of the path loss exponent in Scenario 2 are higher than those obtained in Scenario 1. This may be due to the fact that in Scenario 2 the Tx antenna has a lower height and it is located next to the passenger seats. Thus, MPCs reflected from the seats suffer greater attenuation and the direct path prevails. These results suggest that the path loss behavior is sensitive to the Tx antenna location.

\section{B. Delay spread}

The knowledge of the time dispersion of a channel can be used to improve the design of wireless systems. The rootmean-square (rms) delay spread, denoted by $\tau_{\text {rms }}$, is the most

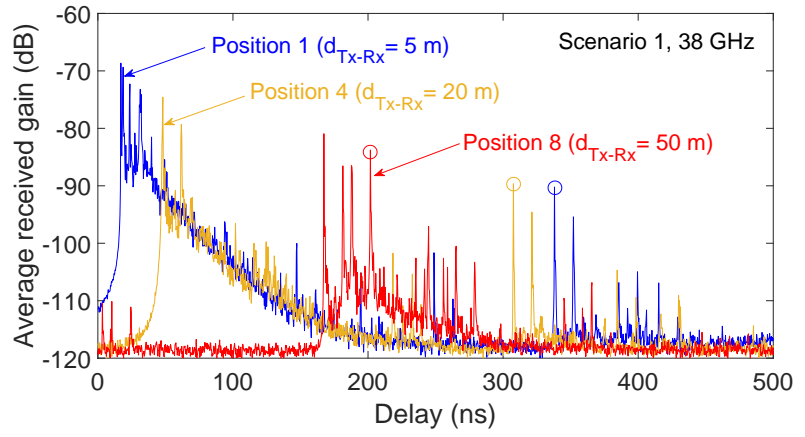

Fig. 6. PDP measured in Scenario 1, positions 1,4 and 8 , in the $38 \mathrm{GHz}$ frequency band. The circle marker corresponds to the reflection from the end of the wagon.

relevant parameter used to describe the time dispersion of wireless channels. $\tau_{\text {rms }}$ is derived as the second-order central moment of the power delay profile (PDP) [19]:

$$
\tau_{r m s}(d) \triangleq \sqrt{\frac{\sum_{n=1}^{N}\left(\tau_{n}-\bar{\tau}(d)\right)^{2} P D P\left(\tau_{n}, d\right)}{\sum_{n=1}^{N} \operatorname{PDP}\left(\tau_{n}, d\right)}},
$$

where $\tau_{n}$ refers to the $\mathrm{n}$-th delay bin. Assuming ergodicity [9], the PDP can be estimated averaging the channel impulse response (CIR) over the $m$ positions of the URA:

$$
\operatorname{PDP}(\tau, d)=E_{m}\left\{|h(\tau, d)|^{2}\right\} .
$$

The CIR is obtained as the inverse Fourier transform of $H(f, d)$. As an example, three PDPs measured in the $38 \mathrm{GHz}$ frequency band (averaged over all $\mathrm{Rx}$ antenna positions in the URA) are shown in Fig. 6 for the Tx antenna positions 1, 4 and 8 in Scenario 1, for a Tx-Rx distance equal to 5, 20 and $50 \mathrm{~m}$, respectively. The shape of the PDPs confirms the great multipath richness in this type of environment. The term $\bar{\tau}$ in (4) represents the mean delay spread introduced by the channel and it is derived from the PDP as

$$
\bar{\tau}(d) \triangleq \frac{\sum_{n=1}^{N} \tau_{n} P D P\left(\tau_{n}, d\right)}{\sum_{n=1}^{N} P D P\left(\tau_{n}, d\right)} .
$$

The values of $\tau_{\mathrm{rms}}$ derived in both scenarios are shown in Fig. 7. A threshold (TH) of $20 \mathrm{~dB}$ and a Hamming windowing method have been considered. The delay spread exhibits a similar behavior in both scenarios. $\tau_{\mathrm{rms}}$ increases to a certain Tx-Rx distance (between 20-40 $\mathrm{m}$ in Scenario 1, and $40 \mathrm{~m}$ in Scenario 2), from which it begins to decrease. Although this behavior is different from the one that appears in other indoor environments, e.g., office and in building, where in general the delay spread has a clear growing trend with the distance, a similar relationship was also observed by other authors. Thus, Bultitude et al. obtained a similar relationship in an empty building [20], and Vitucci et al. in a large indoor environment with highly reflective floors and ceilings [21].

The non-monotonic behavior of $\tau_{\text {rms }}$ can be explained intuitively in the following way. Taking into account that the $\mathrm{Rx}$ antenna is placed near the cabin (there is a separation 

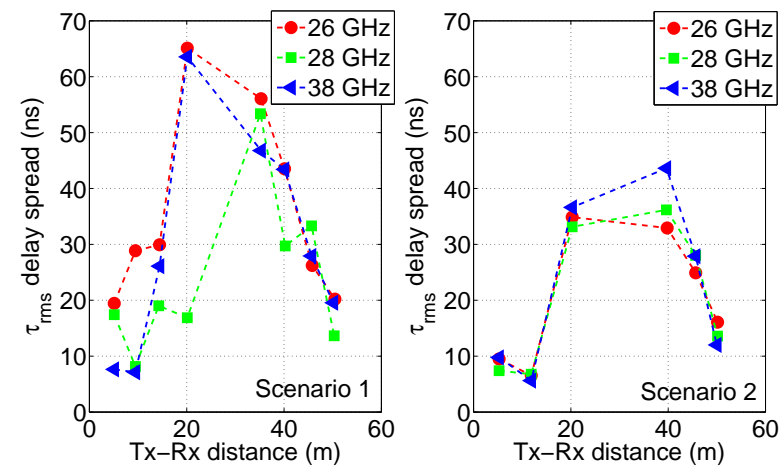

Fig. 7. $\tau_{\text {rms }}$ in terms of the Tx-Rx distance derived for Scenario 1 (left) and Scenario 2 (right).

distance of $2 \mathrm{~m}$ ), and the Tx antenna is moved away from it along the wagon, the relative path length of the MPCs to the direct component, and therefore the relative powers and delays, depend on the Tx-Rx distance. Due to the dimensions and geometry of the wagon, and antennas height, the excess delay associated to the reflections from the ceiling, floor and side walls is small. Nevertheless, the single reflection from the end wall of the wagon, indicated by the circular markers in the PDPs shown in Fig. 6, has a major impact on $\tau_{\text {rms }}$. For small Tx-Rx distances, the relative distance traveled by the MPCs is large compared with the one traveled by the direct component. Therefore, the MPCs have small relative powers compared to the direct component, but large excess delays. In this situation, the single reflection from the end of the wagon has a long delay, about 323 ns relative to the direct component for position 1 at $38 \mathrm{GHz}$, but its power is about $23 \mathrm{~dB}$ lower than the direct component, higher than the $\mathrm{TH}$ level used to derive $\tau_{\text {rms }}$. When the Tx-Rx distance increases up to about $20 \mathrm{~m}$, the relative delay of the reflection from the end of the wagon compared to the direct component is reduced. For example, for the position 4 at $38 \mathrm{GHz}$, the relative delay is about $260 \mathrm{~ns}$, and the relative power is about $15 \mathrm{~dB}$, less than the TH level. At these distances, the reflection from the end of the wagon is taken into account in $\tau_{\text {rms }}$, increasing its value. As the Tx-Rx distance continues to increase, the differential lengths of the MPCs decrease due to the waveguide effect, while at the same time, the relative powers of the MPCs decrease. In this situation, the excess delay of the reflection from the end is about $35 \mathrm{~ns}$, and its relative power is about $3 \mathrm{~dB}$. As a result, $\tau_{\text {rms }}$ decreases.

From Fig. 7, the variation of $\tau_{\mathrm{rms}}$ with the frequency is minimal in Scenario 2, and only appreciable differences appear in Scenario 1 for a Tx-Rx distance less than $40 \mathrm{~m}$. The values of $\tau_{\text {rms }}$ are lower in Scenario 2 due to the greater attenuation of the MPCs reflected from the passenger seats. The values of $\tau_{\text {rms }}$ for both scenarios are summarized in Tables II and III. It is worth noting that the values derived here are larger than those published in the literature for indoor and corridor environments at mmWave frequencies. For example, in indoor environments values of $\tau_{\text {rms }}$ ranging from 8 and $10 \mathrm{~ns}$ have been measured at 28 and $38 \mathrm{GHz}$ in [11], and $17 \mathrm{~ns}$ has been measured at $38 \mathrm{GHz}$ in [12]. In corridor environments, the
TABLE II

Delay SPREAd Values for SCENARIo 1 (IN NANOSECONDS)

\begin{tabular}{l|cccccccc}
\hline \hline & \multicolumn{7}{|c}{ Tx-Rx distance } \\
& $5 \mathrm{~m}$ & $10 \mathrm{~m}$ & $15 \mathrm{~m}$ & $20 \mathrm{~m}$ & $35 \mathrm{~m}$ & $40 \mathrm{~m}$ & $45 \mathrm{~m}$ & $50 \mathrm{~m}$ \\
\hline $26 \mathrm{GHz}$ & 19.45 & 28.86 & 29.92 & 65.09 & 56.06 & 43.41 & 26.21 & 20.19 \\
$28 \mathrm{GHz}$ & 17.41 & 8.15 & 19.01 & 16.87 & 53.37 & 29.72 & 33.30 & 13.64 \\
$38 \mathrm{GHz}$ & 7.63 & 7.11 & 26.09 & 63.54 & 46.78 & 43.41 & 27.29 & 19.56 \\
\hline
\end{tabular}

TABLE III

Delay SpREad Values fOR SCENARIo 2 (IN NANOSECONDS)

\begin{tabular}{l|cccccc}
\hline \hline & \multicolumn{6}{|c}{ Tx-Rx distance } \\
& $5 \mathrm{~m}$ & $12 \mathrm{~m}$ & $20 \mathrm{~m}$ & $40 \mathrm{~m}$ & $45 \mathrm{~m}$ & $50 \mathrm{~m}$ \\
\hline $26 \mathrm{GHz}$ & 9.50 & 6.54 & 34.86 & 32.93 & 24.90 & 16.07 \\
$28 \mathrm{GHz}$ & 7.39 & 6.76 & 33.13 & 36.16 & 28.02 & 13.59 \\
$38 \mathrm{GHz}$ & 9.77 & 5.63 & 36.61 & 43.62 & 27.88 & 12.01 \\
\hline
\end{tabular}

results reported in [22] are lower than $5 \mathrm{~ns}$ in both 28 and $38 \mathrm{GHz}$ frequency bands.

\section{Conclusions}

In this correspondence, we have presented useful measurement results on path loss and delay spread in an intra-wagon environment at mmWave frequencies with applications to the future $5 \mathrm{G}$ systems. The results show that a waveguide effect occurs when the Tx-Rx distance increases. For Scenario 1, values of the path loss exponent lower than free space have been obtained, whereas for Scenario 2 the values are higher due in part to the height and proximity of the Tx antenna to the passenger seats.

The waveguide effect has also been observed in the time dispersion. For Scenario 1, the value of $\tau_{\text {rms }}$ ranges from 7 to $65 \mathrm{~ns}$, whereas for Scenario 2 is lower, ranging from 6 to $43 \mathrm{~ns}$. Note that the values obtained here are significantly higher than those measured in indoor and corridor environments at the same frequency bands, which confirms the great multipath richness in this particular environment due to the presence of numerous metallic elements.

It is evident that the effect of people alters the propagation conditions. In this sense, the results reported here, apart from providing interesting knowledge about the intra-wagon propagation channel characteristics, would allow to calibrate propagation models based on ray-tracing techniques. Then, these models could enable the evaluation of blocking and fading effects of passengers over the received signal.

\section{ACKNOWLEDGEMENT}

The authors would like to thank the staff of FGV who have facilitated the realization of the measurements campaing, in particular Julia Iserte Villalba and Francisco M. Brox López.

\section{REFERENCES}

[1] K. Guan, G. Li, T. Kürner, A. F. Molish, B. Peng, R. He, B. Hui, J. Kim, and Z. Zhong, "On millimeter wave and $\mathrm{THz}$ mobile radio channel for smart rail mobility," IEEE Trans. Veh. Technol., vol. 66, no. 7, pp. 56585674, Jul. 2017.

[2] K. Yang, J.-P. G. M. Berbineau, Y. Cocherill, and D. Seetharamdoo, "Propagation measurements with regional train at $60 \mathrm{GHz}$ for virtual coupling application," in Proc. 11th Euro Conf. Antennas Propag. (EuCAP), Mar. 2017, pp. 126-130. 
[3] World Radiocommunications Conference, "Resolution 238," 2015.

[4] B. Bulut, T. Barratt, D. Kong, J. Cao, A. L. Freire, F. Tila, S. Armour, M. Beach, and A. Nix, "Millimeter wave channel measurements in a railway depot," in Proc. IEEE 28th Апnи. Int. Sy,p. Person. Indoor Mobile Radio Commun. (PIMRC), Oct. 2017, pp. 1-5.

[5] C.-W. Wang, A. Ghazal, B. Ai, Y. Liu, and P. Fan, "Channel measurements and models for high-speed train communications systems: a survey," IEEE Trans. Intell. Transp. Sys., vol. 18, pp. 974-987, Dec. 2017.

[6] D. He, B. Ai, K. Guan, J. García-Loygorri, L. Tian, Z. Zhong, and A. Hrovat, "Influence of typical railway objects in mmwave propagation channel," IEEE Trans. Veh. Technol., vol. 67, pp. 2880-2892, Apr. 2018.

[7] J. García-Loygorri, C. Briso, I. Arnedo, C. Calvo, M. Laso, D. He, F. Jiménez, and V. González, "Wideband channel modeling for mm-wave inside trains for 5G-related applications," Wireless Commun. Mobile Comput., vol. 2018, pp. 1-11, Apr. 2018.

[8] M. G. Sánchez, L. de Haro, A. G. Pino, and M. Calvo, "Human operator effect on wide-band radio channel characteristics," IEEETrans. Antennas Propag., vol. 45, no. 8, pp. 1318-1320, August 1997.

[9] A. F. Molisch, Wireless Communications, 2nd ed. Wiley-IEEE Press, 2010.

[10] R. Steele and L. Hanzo, Mobile radio communications, 2nd ed. WileyIEEE Press, 1999.

[11] J. Huang, C.-X. Wang, R. Feng, W. Zhang, and Y. Yang, "Multifrequency mmwave massive MIMO channel measurements and characterization for 5G wireless communications systems," IEEE J. Sel. Areas Commun., vol. 35, pp. 1591-1605, Jul. 2017.

[12] G. R. MacCartney, T. S. Rappaport, S. Sun, and S. Deng, "Indoor office wideband millimeter-wave propagation measurements and channel models at $28 \mathrm{GHz}$ and $73 \mathrm{GHz}$ for ultra-dense $5 \mathrm{G}$ wireless networks (invited paper)," IEEE Access, vol. 3, pp. 2388-2424, Dec. 2015.

[13] 3GPP TR 25.996, "Spatial channel model for multiple input multiple output (MIMO) simulations," Sept. 2012.
[14] N. O. Oyie and T. J. O. Afullo, "Measurements and analysis of largescale path loss model at 14 and $22 \mathrm{GHz}$," IEEE Access, vol. 6, pp. 17 205-17 214, Jan. 2018.

[15] S. Deng, M. K. Samimi, and T. S. Rappaport, " $28 \mathrm{GHz}$ and 73 $\mathrm{GHz}$ millimeter-wave indoor propagation measurements and path loss models," in IEEEInternational Conference on Communications, June 2015, pp. 1244-1250.

[16] N. Blaunstein, "Average field attenuation in the nonregular impedance street waveguide," IEEETrans. Antennas Propag., vol. 46, no. 12, pp. 1782-1789, December 1998.

[17] S. Geng and P. Vainikainen, "Millimeter-wave propagation in indoor corridors," IEEE Antennas Wireless Propag. Lett., vol. 8, pp. 1242-1245, Nov. 2009.

[18] I. S. Batalha, A. V. R. Lopes, J. P. L. Araújo, B. L. S. Castro, F. J. B. Barros, G. P. D. S. Cavalcante, and E. G. Pelaes, "Indoor corridor office propagation measurements and channel models at 8, 9, 10 and $11 \mathrm{GHz}$," IEEE Access, vol. 7, pp. $55005-55021$, May. 2019.

[19] J. D. Parsons, The mobile radio propagation channel, 2nd ed. Wiley, 2000.

[20] R. Bultitude, P. Melançon, H. Zaghloul, G. Morrison, and M. Prokki, "The dependence of indoor radio channel multipath characteristics on transmit/receive ranges," IEEE J. Sel. Areas Commun., vol. 11, no. 7, pp. 979-990, September 1993.

[21] E. M. Vitucci, F. Fuschini, and V. Degli-Esposti, "Ray tracing simulation of the radio channel time- and angle-dispersion in large indoor environments," in European Conference on Antennas and Propagation, Apr. 2014, pp. 1771-1774.

[22] A. M. Al-samman, T. A. Rahman, and M. H. Azmi, "Indoor wideband radio propagation measurements and channel models for $5 \mathrm{~g}$ millimetre wave wireless communications at $19 \mathrm{GHz}, 28 \mathrm{GHz}$, and $38 \mathrm{GHz}$ bands," Wireless Communications and Mobile Computing, pp. 1-12, 2018. 